\title{
Distribuição espacial de focos do Aedes Aegypti em Campina Grande, Paraíba
}

A proliferação do mosquito Aedes aegypti tem aumentado nos últimos anos nos aglomerados urbanos, ocasionando sucessivos episódios de epidemias por todo o país. Alguns fatores vêm contribuir para a proliferação do mosquito Aedes aegypti, tais como a escassez hídrica e a deficiência da cobertura da rede de abastecimento. Diante do exposto, o presente trabalho teve como objetivo analisar a distribuição espacial de focos do Aedes aegypti nos bairros de Campina Grande/PB, em decorrência das falhas do fornecimento de água. A metodologia de pesquisa utilizada neste trabalho está fundamentada em um estudo epidemiológico com delineamento ecológico e abordagem quantitativa. Por meio da análise espacial se observou autocorrelação espacial negativa e positiva em alguns bairros de Campina Grande. A auto correlação espacial positiva alerta para o aumento dos índices de larvas do Aedes aegypti em determinado bairros. Fato que resulta da permanência de depósitos ao nível do solo, utilizados para armazenamento de água. Neste sentido, observa-se que a visualização dessas localidades evidenciam a vulnerabilidade ambiental dessas áreas, e a probabilidade da população destes bairros estarem suscetíveis a doenças transmitidas pelo Aedes aegypti. Assim, ressalta-se a necessidade de investir na gestão eficiente do uso das águas do açude Epitácio Pessoa, consequentemente garantindo a regularização do abastecimento sem que haja necessidade de estocagem de água.

Palavras-chave: Dengue; Escassesz hídrica; Geoestatística.

\section{Spatial distribution of Aedes Aegypti focus in Campina Grande, Paraíba}

\begin{abstract}
The proliferation of Aedes aegypti mosquito has increased in recent years in urban agglomerations, causing successive episodes of epidemics throughout the country. Some factors contribute to the proliferation of the Aedes egypti mosquito, such as water scarcity and lack of coverage from supply network. Given the above, this work aimed to analyse the spatial distribution of Aedes Egypti focus in the neighbours of Campina Grande/PB, due to the failures in water supply networks. The research methodology used in this work is based in a epidemiological study with an ecological outline and a quantitative approach. Through spatial analysis it was observed a negative and positive spatial autocorrelation in some of the neighbours of Campina Grande. The positive spatial autocorrelation warns to an increase of indications of Aedes aegypti larvas in specific neighbours. This fact is a result from the permanence of ground-level deposits used for wate storage. In this sense, it is observed that the visualization of these locations shows the environmental vulnerability of these areas, and the probability of the population of these neighborhoods being susceptible to diseases transmitted by Aedes aegypti. Therefore, the need to invest in the efficient management of the water use of the Epitácio Pessoa reservoir is emphasized, thus ensuring the regularization of supply without the need for water storage.
\end{abstract}

Keywords: Dengue; Water scarcity; Geostatistics.

Topic: Epidemiologia e Saúde Ambiental

Reviewed anonymously in the process of blind peer
Received: 02/10/2019

Approved: 26/11/2019
Luana Andrade Lima Querino (iD)

Universidade Federal de Campina Grande, Brasil

http://lattes.cnpq.br/3979403454435718

http://orcid.org/0000-0001-5874-6006

luanaandradelima@gmail.com

Patrício Marques Souza (D)

Universidade Federal de Campina Grande, Brasil

http://lattes.cnpq.br/0395671025242338

http://orcid.org/0000-0002-2064-3818

patriciomsouza@gmail.com

Ricardo Alves de Olinda (it)

Universidade Estadual da Paraíba, Brasil

http://lattes.cnpq.br/7767223263366578

http://orcid.org/0000-0002-0509-8428

ricardo.estat@yahoo.com.br
Juliana Meira de Vasconcelos Xavier (iD)

Universidade Federal de Campina Grande, Brasil

http://lattes.cnpq.br/2221607460684235

http://orcid.org/0000-0003-0603-1943

juliana-mvasconcelos@hotmail.com

DOI: 10.6008/CBPC2179-6858.2019.006.0010

Referencing this:

QUERINO, L. A. L.; SOUZA, P. M.; OLINDA, R. A.; XAVIER, J. M. V.. Distribuição espacial de focos do Aedes Aegypti em Campina Grande, Paraíba. Revista Ibero Americana de Ciências Ambientais, v.10, n.6, p.101-113, 2019. DOI: http://doi.org/10.6008/CBPC21796858.2019.006.0010 


\section{INTRODUÇÃO}

Atualmente a dengue é um dos principais agravos que acomete a saúde pública no Brasil. Em meados de 2014, o país vivenciou o início uma epidemia de dengue e um rápido processo de introdução de dois novos arbovírus, chikungunya e zika também transmitidos pelo mosquito Aedes aegypti (NUNES et al., 2014). No ano de 2015 foram registrados 1.485 .397 casos de dengue no Brasil, a região nordeste registrou $(18,1 \%)$ desse total, sendo o segundo maior número de casos notificados (BRASIL, 2015). De acordo com o boletim epidemiológico do Estado da Paraíba, em 2015 o Estado registrou um acréscimo de 303,28\% nos casos de dengue em relação ao ano anterior (PARAÍBA, 2015).

Condições ambientalmente favoráveis contribuem para a adaptação e propagação do mosquito Aedes aegypti, permitindo sua rápida difusão espacial e acentuando episódios de epidemias nas áreas urbanas (DU et al., 2019; HACKL et al., 2019; NEIDERUD, 2015). Condições precárias de saneamento e infraestrutura inadequada no abastecimento das cidades, são fatores que possivelmente explicam a dificuldade no controle da dengue e outras viroses transmitidas pelo mosquito Aedes aegypti.

Nesse contexto, em virtude da ausência de chuvas e a escassez hídrica, Campina Grande enfrentou graves problemas no abastecimento, o reservatório que abastece a cidade atingiu o volume morto e a qualidade da água se apresentava e comprometida. Uma resposta emergencial a escassez hídrica, foi o racionamento anunciado no ano de 2014 pela Companhia de Água e Esgotos da Paraíba - CAGEPA, com um cronograma de rodízios alternando os dias de fornecimento (GRANDE et al., 2016; RÊGO et al., 2015).

A precariedade no abastecimento leva à adoção de práticas de estocagem de água em recipientes (baldes e tonéis) que por sua vez contribuem para a positividade e produtividade do mosquito Aedes aegypti (CORTE et al., 2018; CONYER et al., 2018; FERDOUSI et al., 2015; GIL et al., 2015; TSUZUKI et al., 2009). De modo geral ao tentar resolver a falha do fornecimento de água, armazenando-a nos domicílios para enfrentar a crise hídrica, surge um novo problema que deve ser encarado como uma ameaça as ações de controle ao mosquito Aedes aegypti, cujo objetivo se baseia principalmente na eliminação acúmulo de água nos domicílios. Diante dos fatos expostos, o presente trabalho teve como objetivo identificar os depósitos que caracterizam o índice de infestação de larvas do mosquito Aedes aegypti e a distribuição espacial de focos nos bairros de Campina Grande/Paraíba.

\section{MATERIAIS E MÉTODOS}

O presente trabalho foi realizado na cidade de Campina Grande, localizada na Mesorregião do Agreste Paraibano. Situa-se a 70 13'11" de latitude Sul e a 35 52'31" de longitude Oeste, possuindo uma área territorial de aproximadamente $621 \mathrm{~km}^{2}$, e com população estimada em 410.332 habitantes (IBGE, 2017). O município de Campina Grande é a segunda maior economia do estado da Paraíba, o PIB - Produto Interno Bruto da cidade registrou em 2014 um crescimento de 14,7\%. Sendo portanto, o maior centro econômico do interior do estado, em decorrência das atividades do comércio, atividades educacionais de nível superior, polos tecnológicos na área de couro, calçados e tecnologia da informação (PARAÍBA, 2014). 
O sistema atual de abastecimento de água em Campina Grande e em outras 18 cidades depende do reservatório Epitácio Pessoa, também conhecido popularmente como Açude Boqueirão. O reservatório está localizado no município de Boqueirão, a aproximadamente $40 \mathrm{~km}$ de distância da cidade de Campina Grande, está inserido na Bacia do rio Paraíba, a bacia de drenagem da barragem está localizada em região semiárida com taxas de evaporação muito altas (BRAGA et al., 2001). Atualmente é um dos principais reservatórios de captação de água de chuva do estado da Paraíba, com capacidade de armazenamento superior a 411 milhões de metros cúbicos.

A metodologia de pesquisa utilizada neste trabalho está fundamentada em um estudo epidemiológico de caráter ecológico e abordagem quantitativa. O estudo ecológico investiga questões de saúde ambiental, comparando populações em uma série temporal utilizando dados já existentes de fontes secundárias (BONITA et al., 2010; ROUQUAYROL et al., 2006).

Os dados utilizados neste trabalho foram obtidos na Secretaria de Vigilância Ambiental de Campina Grande/Paraíba . O conjunto de dados é referente a documentos oficiais do LIRAa (Levantamento Rápido de Índices para Aedes aegypti) relativos aos 51 bairros da cidade de Campina Grande, correspondente ao período de Janeiro de 2014 a Dezembro de 2017. O LIRAa é um programa utilizado pelo Ministério da Saúde na Vigilância Entomológica do Aedes aegypti no Brasil (BRASIL, 2009). Possibilita o levantamento de informações, as quais foram utilizadas neste trabalho, sobre o IP- Infestação Predial, que indicam risco de epidemia para determinada área, como também o ITR- índice por tipo de recipiente, que caracteriza os tipos de depósitos positivos com larvas do Aedes aegypti.

O ITR identifica os tipos de depósitos que podem se tonar criadouros para o mosquito: A1 depósito de água elevado, ligado à rede pública ou sistema de captação mecânica em poço; A2 depósitos ao nível do solo para armazenamento doméstico (tonel, tambor, barril, depósitos de barro); B depósitos móveis (vasos, pratos, garrafas retornáveis); C depósitos fixos (calhas, lajes, ralos, sanitários em desuso, piscinas não tratadas, fontes ornamentais, etc.); D1 (pneus e outros materiais rodantes); D2 resíduos sólidos (recipientes plásticos, garrafas PET, latas, sucatas, etc.).

\section{Análise estatística dos dados}

Com o intuito de caracterizar os tipos de depósitos identificados com larvas do mosquito Aedes aegypti nos bairros de Campina Grande, utilizou-se o ITR. Os dados foram tabulados por ano de estudo mediante o uso de porcentagem. As autocorrelações espaciais foram identificadas por meio das técnicas da estatística espacial para dados de área. Sendo assim, para verificar a estrutura da autocorrelação espacial do IP entre os bairros da cidade de Campina Grande, foram calculados os índices de Moran Global e Local. Na sequência, os resultados foram apresentados através do diagrama de espalhamento de Moran, Moram Map e Lisa Map, adotando-se os níveis de significância de 5\% e 1\%.

\section{RESULTADOS E DISCUSSÃO}

A seguir se observa o percentual do ITR em Campina Grande no ano de 2014 a 2017. A partir da 
análise do ano de 2014 se obtiveram os seguintes resultados. Os depósitos do tipo A2 (tonel, tambor, barril, baldes, etc) foram os mais frequentes com $52 \%$ identificados com larvas de Aedes aegypti, seguido de $\mathrm{A} 1$ (caixas d'água, etc.) com 38\%, B (vasos, garrafas retornáveis, etc.) 4\%, D1 (pneus e outros materiais rodantes) com \%2, D2(resíduos sólidos) \%2, C (calhas, lajes e toldos em desnível e ralos) 1\%, E (axilas de folhas, buracos em árvores, rochas, restos de animais) com percentual de $1 \%$.

A amostragem para ano de 2015, observou-se um aumento significativo do porcentual de depósitos de armazenamento ao nível do solo em comparação ao ano anterior. Verificou-se que $89 \%$ dos locais onde foram encontrados a presença de larvas de Aedes aegypti, ocorreu nos depósitos do tipo A2 (tonel, tambor, barril, baldes, etc.), D2 (resíduos sólidos) 4\%, B (Vasos, garrafas retornáveis etc.) com 3\%, percebe-se a diminuição da preferência por depósitos do tipo A1 (caixas d'água) apresentando um percentual de apenas $2 \%$, os depósitos do tipo C (calhas, lajes e toldos em desnível, ralos) e D1 (pneus e outros materiais rodantes) apresentaram apenas o percentual de $1 \%$ cada.

Tendo em vista o agravamento da situação hídrica do reservatório Epitácio Pessoa no ano de 2015, inicia-se em Campina Grande um plano de racionamento. Diante desta realidade a população se vê obrigada a armazenar água para suprir as necessidades diárias. Deve-se considerar que, diante desta situação os depósitos utilizados para armazenar água tornaram-se potenciais criadouros do mosquito. Alguns trabalhos evidenciaram uma relação positiva entre a falta de cuidados com os depósitos utilizados para armazenamento de água, quando não devidamente fechados, tornam-se acessíveis à fêmea do mosquito Aedes aegypti para postura dos seus ovos (CHUMSRI, 2018; POWELL et al., 2013; SCANDAR, 2007).

Em estudo realizado em Recife/PE por Bonat et al. (2009), os autores utilizaram armadilhas (ovitrampas) nos domicílios que também tinham recipientes utilizados para armazenamento de água e não estavam devidamente fechados. Entretanto, o estudo demonstrou que os mosquitos mostraram preferência em colocar os ovos em tanques, caixas d'água e tonéis sem tampa diminuindo assim a ocorrência de ovos nas armadilhas.

No ano de 2016, os recipientes que se destacaram com maior frequência foram os depósitos do tipo A2 (tonel, tambor, barril, etc.) com 90\% dos achados de larvas de Aedes aegypti, os depósitos do tipo B (Vasos, garrafas retornáveis etc.) com 5\%, A1(caixas d'água) 2\%, os depósitos do tipo D1 (pneus e outros materiais rodantes), D2 (resíduos sólidos) e C (calhas, lajes e toldos em desnível, ralos etc.) apresentaram apenas $1 \%$ para cada categoria.

Observa-se que os tipos de recipientes preferíveis para a oposição se caracterizam por recipientes para armazenamento de água ao nível do solo, esse fato se dar também pela oferta desses tipos de depósitos. Tendo em vista que diante de uma situação de escassez hídrica a população tende a aumentar o número de depósitos nos seus domicílios, fato também verificado por Grande et al. (2016), em pesquisa realizada sobre o impacto do racionamento de água em Campina Grande, usuários afirmaram aumentar o número de recipientes para armazenar água.

No ano de 2017 a maior incidência de larvas ocorreu nos depósitos do tipo A2 (tonel, tambor, barril, etc.) com 93\%, depósitos do tipo B (Vasos, garrafas retornáveis etc.) 5\%, os depósitos do tipo Ce D2 
apresentaram o mesmo percentual 1\% cada e os demais depósitos A1, D1 e E não pontuaram. Observa-se a tendência ascendente do aumento percentual dos depósitos A2 (tonel, tambor, barril) durante os anos estudados, ou seja, a cada ano ocorre o aumento da proliferação do mosquito em depósitos de armazenamento baixo. Os resultados refletem os efeitos da intensificação da escassez hídrica e da prolongação dos dias sem água em Campina Grande. Com o passar dos anos a oferta se tornava mais escassa e a alternativa utilizada pela população se concentrava na utilização de depósitos para armazenamento de água. O controle da população de Aedes aegypti se baseia na eliminação de criadouros, evitando sua proliferação.

A seguir será analisado para cada ano os testes do Moran Map, Lisa Map e espalhamento de Moran, para a análise do índice de Infestação Predial em Campina Grande. A análise no Moran Map foi realizada com base nos ciclos de cada ano estudado, que obtiveram dependência espacial positiva dada por valor-p significativo $(<0,001)$. A aplicação do Moran Map e Lisa Map buscou analisar a existência da autocorrelação espacial entre os bairros. Sendo possível observar no Moran Map quais os bairros influenciaram positivamente para proliferação de larvas do Aedes aegypti em Campina Grande, como também os bairros que influenciaram para diminuição dos índices, ambas hipótese confirmados pelo espalhamento de Moran.

Foram levados em consideração no Lisa Map os bairros que possuem estrutura de autocorrelação espacial significativa positiva ou negativa, que apresentaram significância abaixo de 5\%, com intervalos entre $(5 \%, 1 \%, 0,1 \%)$ respectivamente. A seguir se apresenta os resultados para a análise da autocorrelação espacial do 10 LIRAa do ano de 2014. Diante das análises realizadas, verificou-se a influência espacial entre bairros de Campina Grande expressos nos resultados a seguir.

Observa-se no mapa (Figura 1A) a análise referente ao Moran Map, a formação de aglomerados de bairros que possuem estrutura de autocorrelação espacial significativa, desta forma influenciando positivamente para o aumento dos índices nos bairros: Santo Antônio; José Pinheiro; Monte Castelo; Serrotão e Castelo Branco. Conforme também ratificado pelo diagrama de espelhamento (Figura 1C) a influência dos bairros: José Pinheiro; Santo Antônio e Monte Castelo. Estes bairros se apresentam influentes com percentuais de significância positiva de 0,1\% apontados pelo no Lisa Map (Figura 1 B).

Estes bairros são ainda mais influentes para o aumento das médias espaciais e contribuem significativamente para o aumento do índice larvário de Aedes aegypti, contribuindo também para a positividade dos bairros vizinhos. Exercendo função inversa, ou seja, contribuindo para a diminuição da propagação do Aedes aegypti, estão os bairros do São José, Prata e Estação Velha, estes bairros apresentaram valores negativos e contribuem para a diminuição dos índices.

Conforme análise do 2 LIRAa de 2014 (Figura 2A) os bairros: Mirante; Vila Cabral; Sandra Cavalcante; Itararé; Bodocongó; Novo Bodocongó; Ramadinha; Malvinas e Serrotão se apresentam exercendo influência positiva para o aumento dos índices larvários. O diagrama de espelhamento (Figura $2 \mathrm{C}$ ) se destaca também a influência dos bairros: Novo Bodocongó e Ramadinha para a manutenção do Aedes aegypti na cidade de Campina Grande. 


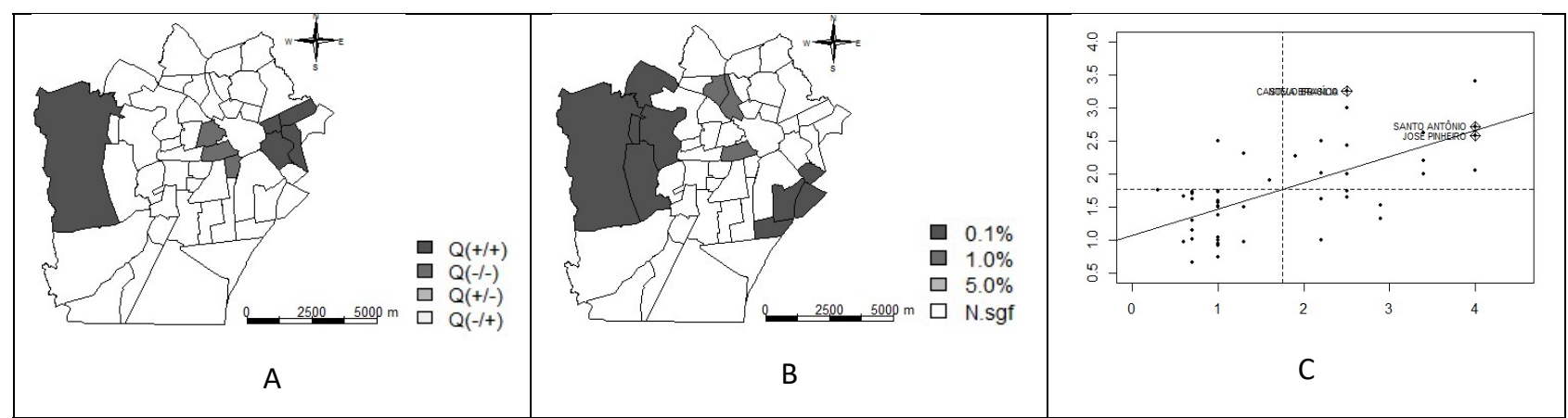

Figura 1: Visualização no Moran Map dos bairros que possuem autocorrelação espacial no 10 LIRAa de 2014(A); ocorrência de bairros significativos apresentados pelo Lisa Map (B); diagrama de espalhamento de Moran (C).

De acordo o Lisa Map (Figura 2B), observa-se alto nível de correlação positiva, com significativa de $0,1 \%$ nos bairros: Vila Cabral; Sandra Cavalcante; Bodocongó; Malvinas e Serrotão. Essa autocorrelação espacial se dar nos bairros que apresentam influência positiva e contribuem para o aumento das médias do LIRAa.

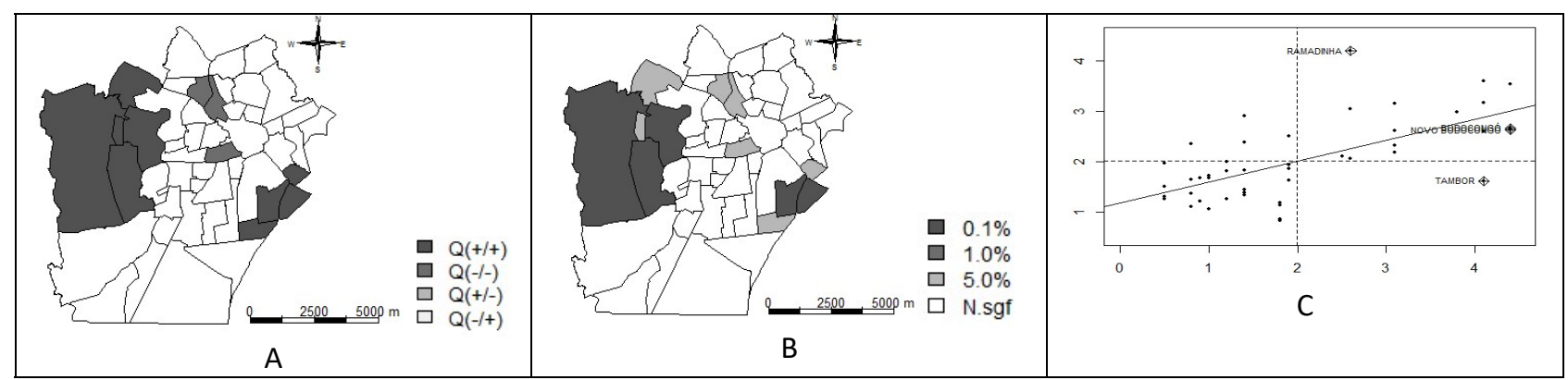

Figura 2: Visualização no Moran Map dos bairros que possuem autocorrelação espacial no 2ㅇ LIRAa de 2014(A); ocorrência de bairros significativos apresentados pelo Lisa Map (B); diagrama de espalhamento de Moran (C).

Os bairros Jeremias, Palmeira e São José (Figura 2 B) se apresentam significativos para diminuição das incidência larvária de Aedes aegypti, como também não oferecem risco aos bairros vizinhos. Apresentase na Figura 3A, a análise do Moran Map para 3은 LIRa de 2014. Foram identificados com influência positiva na infestação larvária do Aedes aegypti os bairros a seguir: Alto Branco; Nações; Jardim Tavares; Castelo Branco; Santo Antônio; José Pinheiro; Mirante; Monte Castelo e Nova Brasília. Podemos destacar ainda no Lisa Map (Figura 3B). Alguns bairros apresentam autocorrelação altamente significativa positiva com percentual de 0,1\% são eles: Jardim Tavares; Castelo Branco; Nova Brasília e Monte Castelo. Estes bairros apresentam influência positiva contribuindo para o aumento das médias do índice de infestação e consequentemente influenciam para o aumento de focos do mosquito nos bairros vizinhos.

Em situação inversa conforme apresentados no Moran Map (Figura 3A) estão os bairros: Universitário; Monte Santo; Bela Vista; Prata; Pedregal e Centenário que apresentam médias negativas e influenciam os bairros vizinhos para a diminuição da incidência do Aedes aegypti. Os bairro apontados no Moran Map (Figura 3A) com exceção do bairro Monte Santo, foram apontados também pelo Lisa Map (Figura 3B) sendo altamente significativos neste ciclo com $0,1 \%$ de significância, contribuindo desta forma para diminuição da incidência de focos do mosquito Aedes aegypti. Ainda de acordo com o espalhamento de Moran (Figura 3C) se observa o bairro do Pedregal, com a diminuição dos índices de infestação larvária 
neste ciclo.

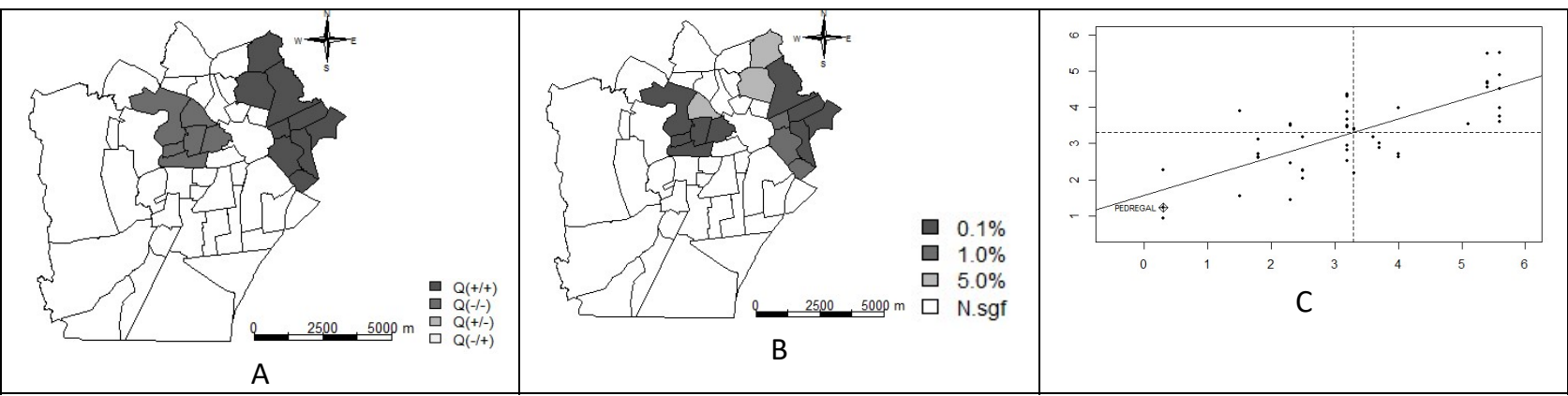

Figura 3: Visualização no Moran Map dos bairros que possuem autocorrelação espacial no 3 LIRAa de 2014(A); ocorrência de bairros significativos apresentados pelo Lisa Map (B); diagrama de espalhamento de Moran (C).

Em busca de melhor compreender a permanência de áreas de influência da manutenção de focos do Aedes aegypti em Campina Grande, as questões referentes ao abastecimento de água e condições de infraestrutura são pertinentes nas discussões deste trabalho. O reservatório Epitácio Pessoa que abastece Campina Grande, passou entre 2014 e 2015 por uma redução considerável em 40\% do seu volume, prosseguindo em declínio nos anos seguintes até atingir o volume morto. Pelos motivos expostos, a população de Campina Grande foi submetida a um sistema de racionamento com períodos de desabastecimento de até 4 dias, de forma alternada nas diversas zonas da cidade (MEDEIROS, 2017).

A crise da água se refere mais do que a escassez hídrica, mas uma crise de gestão (DALEZIOS et al., 2018; SRINIVASAN et al., 2012). De acordo com estudos realizados sobre a gestão do Epitácio Pessoa, a crise do manancial não está pautada nos fatores hidrometeorológicos, mas tem sido apontado falhas, negligências em decisões tardias e equivocadas, ao invés de medidas efetivas para promoção de uma gestão eficaz, em vista da utilização de seus recursos hídricos sem expô-lo a ameaças de racionamento ou a falta de água (GRANDE et al., 2016; RÊGO et al., 2015).

Assim, infere-se que o período de escassez hídrica observado nos últimos anos em Campina Grande e a gestão inadequada do reservatório Epitácio Pessoa, contribuíram para a redução da disponibilidade de água, consequentemente levando a população a manter reservas de depósitos com água nos domicílios. Encontra-se na Figura 4, as análises referentes ao 3 e 4 ㅇ ciclo do LIRAa do ano de 2015, tendo em vista que apenas estes ciclos apresentaram valores significativo de valor-p $(<0,001)$.

Observa-se no 3ㅇ LIRAa de 2015 (Figura 4A) os bairros: Cuité; Jeremias; Palmeira; Monte Santo; Bela Vista; Prata e Centro apresentaram autocorrelação espacial significativa negativa. Estes bairros favorecem a diminuição dos índices, ainda na mesma análise com o diagrama de espalhamento de Moran (Figura 4C) o bairro do Centro, apresenta-se altamente significativo, com índice de autocorrelação espacial com tendência para diminuição dos focos do mosquito. Na análise do Lisa Map os bairros da Palmeia e Prata também se apresentam altamente significativo com $0,1 \%$, conforme verificado (Figura 4B).

Em relação a influência espacial positiva os índices tendem a aumentar nos bairros: Malvinas, Santa Cruz e Cruzeiro conforme observado no Moran Map, Figura 4A. Na análise do Lisa Map (Figura 4B), o bairro Santa Cruz, apresenta-se ainda mais influente com 0,1\% de significância. Conforme podemos observar este bairro contribui espacialmente para aumento de focos do mosquito Aedes aegypti. Assim também verificado 
no diagrama de espalhamento (Figura 4C) a contribuição do bairro Santa Cruz e Presidente Médici para aumento dos índices.

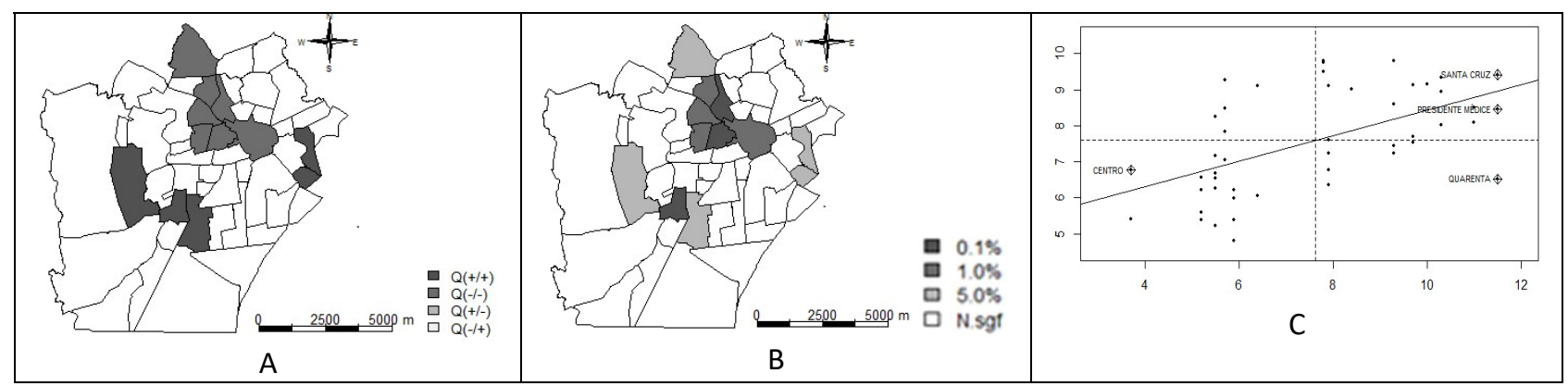

Figura 4: Visualização no Moran Map dos bairros que possuem autocorrelação espacial no 3ㅇ LIRAa de 2015(A); ocorrência de bairros significativos apresentados pelo Lisa Map (B); diagrama de espalhamento de Moran (C).

A seguir na Figura 5A, observa-se com a análise do Moran Map, a influência positiva dos bairros: Castelo Branco, Santo Antonio, José Pinheiro, Monte Castelo e Mirante para o aumento dos focos do mosquito Aedes aegypti no 40 ciclo do LIRAa de 2015. Conforme também pode ser verificado no diagrama de espalhamento (Figura 5C), a contribuição de todos os bairros supramencionados para o aumento dos focos. Neste ciclo, o bairro Monte Santo apresentou significância de $0,1 \%$, ou seja, estava mais propenso a influenciar os bairros vizinhos para aumento das médias do IP (Figura 5B).

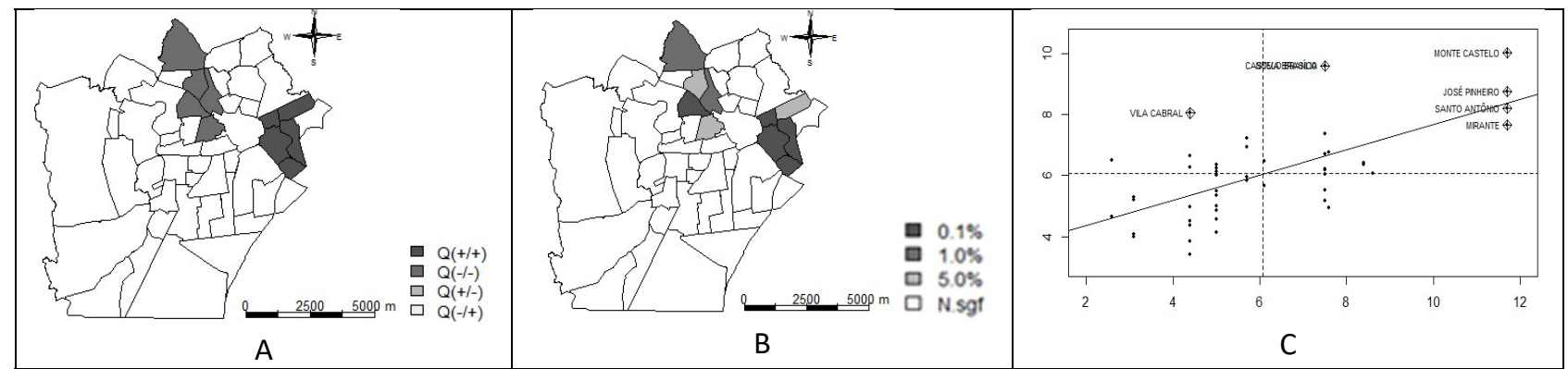

Figura 5: Visualização no Moran Map dos bairros que possuem autocorrelação espacial no 4ㅇ LIRAa de 2015(A); ocorrência de bairros significativos apresentados pelo Lisa Map (B); diagrama de espalhamento de Moran (C).

Ainda de acordo com o 4ํ ciclo do LIRAa de 2015 os bairros: Cuités; Jeremias; Palmeira; Monte Santo e Prata (Figura 5A), estes bairros que apresentaram expressividade negativa na autocorrelação espacial e favorecem a diminuição dos focos do mosquito. O bairro do Monte Santo se apresenta ainda mais significativo, conforme podemos visualizar no Lisa Map (Figura 5B) com percentual de 0,1\%, contribuindo para a diminuição dos índices de infestação lavaria.

Uma das averiguações realizadas pelo LIRAa consiste em identificar os locais de reprodução das larvas do Aedes aegypti. Em Campina Grande foi verificado que os recipientes onde foram encontradas larvas do mosquito, são os mesmos utilizados para armazenamento de água no ambiente domiciliar, que nos faz evidenciar a problemática estrutural no abastecimento na cidade.

A ausência da cobertura de saneamento é um dos principais motivos que leva a população a necessitar armazenar água em tonéis, tambores, baldes, etc.. Estudo conduzido por Dari (2015) em Campina Grande, verificou alguns pontos da cidade sem atendimento do sistema de abastecimento. E ainda de acordo com o autor supramencionado, a cobertura de rede de água não é a garantia da prestação do serviço com 
qualidade para o usuário, uma vez que não assegura disponibilidade de água de modo contínuo e ininterrupto.

Resultados semelhantes foram observados por Oliveira (2018), ao comparar a situação de abastecimento pela rede pública nas macrorregiões da Paraíba, a pesquisa evidenciou que Campina Grande apresenta os piores percentuais de cobertura em toda a série histórica, mas com um crescimento lento ascendente. Teixeira (2014) salienta que o saneamento promove a prevenção de doenças, por meio da interrupção de algumas das vias de transmissão. Essa perspectiva também foi verificada por Johansen (2014) em Caraguatatuba/SP, ao constatar autocorrelação espacial positiva na incidência de dengue nas localidades com piores condições de acesso a água.

Já na Figura 6 e 7, apresentamos os resultados do 2 e 3 LIRAa do ano de 2016. Para o 2 LIRAa, diante da análise do Moran Map (Figura 6 A) os bairros: Tambor, Itararé, Distrito Industrial, Velame, Jeremias e Palmeira apresentaram médias negativas influenciando os bairros vizinhos a manterem seus índices baixos. Neste ciclo o bairro da Palmeira (Figura 6B) se destacou com percentual de 0,1\%, apresentando-se altamente significativo para diminuição dos valores do IP. Com influência espacial positiva para permanência de focos do mosquito e influenciar os bairros vizinhos estão os bairros: Jardim Tavares, Castelo Branco e Monte Castelo(Figura 6A).

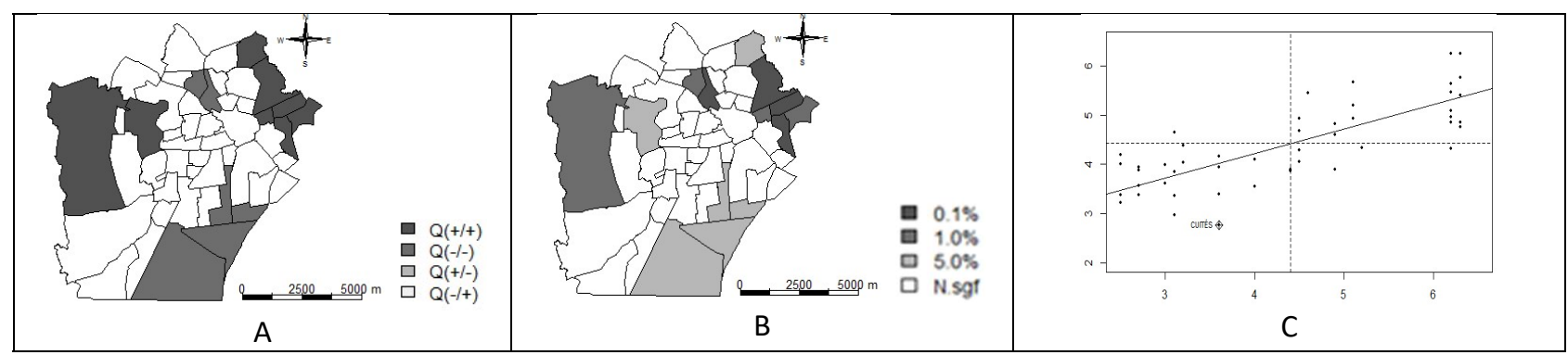

Figura 6: Visualização no Moran Map dos bairros que possuem autocorrelação espacial no 2ㅇ LIRAa de 2016 (A); ocorrência de bairros significativos apresentados pelo Lisa Map (B); diagrama de espalhamento de Moran (C).

A seguir na Figura 7 se apresenta o resultado para o 3 ㄴ LIRAa de 2016. Os bairros que apresentaram influência positiva no 3o LIRAa (Figura 7A) foram: Novo Bodocongó; Monte Castelo; Castelo Branco; Santo Antônio; José Pinheiro; Mirante e Monte Castelo. De acordo com o Lisa Map (Figura 7B), estes bairros apresentam o percentual de significância 0,1\%, conforme pode ser verificado no Moran Map (Figura 7A ) e no diagrama de espalhamento (Figura 7C). Contribuindo desta forma para o aumento da incidência larvária do mosquito Aedes aegypti nestes bairros como também nos bairros vizinhos, ou seja, são altamente significativos para aumento dos índices de IP no 3o ciclo de 2016. Entretanto os bairros: Santo Antônio; José Pinheiro; Mirante e Monte Castelo se apresentaram influentes negativamente, são bairros que espacialmente não contribuem para manutenção de focos do mosquito.

Acredita-se que uma das possíveis justificativas para a dispersão dos focos do Aedes aegypti nos bairros de Campina Grande, ocorra devido a recipientes improvisados, sem tampas e sem vedação correta, que são utilizados para armazenamento de água nos domicílios principalmente em períodos de racionamento. 


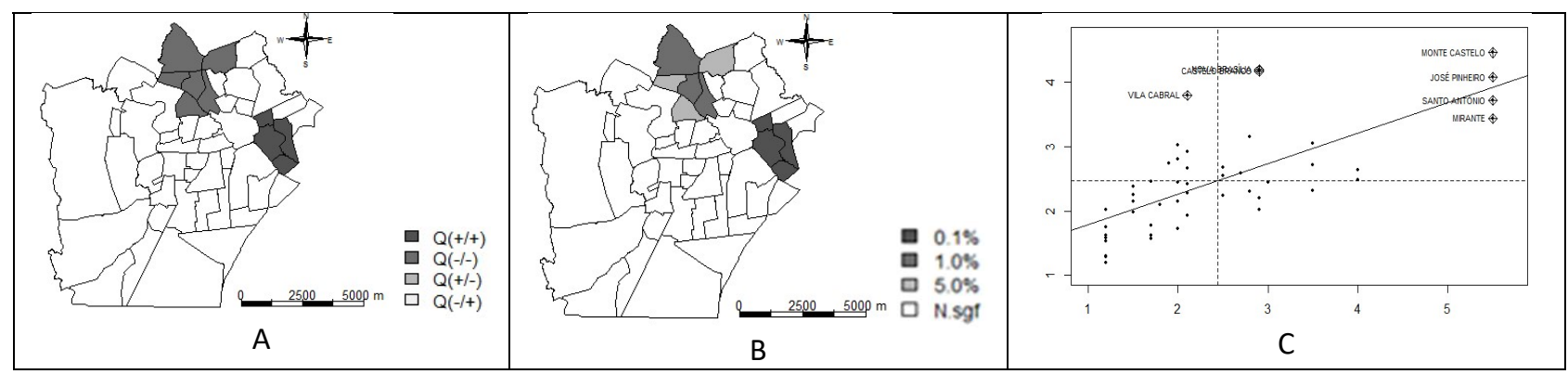

Figura 7: Visualização no Moran Map dos bairros que possuem autocorrelação espacial no 3 LIRAa de 2016 (A); ocorrência de bairros significativos apresentados pelo Lisa Map (B); diagrama de espalhamento de Moran (C).

Nesse sentido, alguns trabalhos corroboram para a evidência da relação positiva entre o acumulo de água no ambiente domiciliar e a manutenção de criadouros do mosquito (CABRAL et al., 2012; SCANDAR et al., 2010; YUE et al., 2018). Conforme também verificado por Tsuzuki e colaboradores (2009), em áreas urbanas da cidade de Ho Chi Minh no Vietnã e por Gonzalez (2017) em bairros com altos índices de dengue na Venezuela, ambos trabalhos verificaram que a associação entre a abundância de larvas do Aedes aegypti está diretamente relacionada com a deficiência no sistema de abastecimento de água e o uso de tonéis para armazenamento. $\mathrm{O}$ acesso ao um sistema de abastecimento de água que garanta continuidade dos serviços sem interrupção, pode levar a uma redução ou tornar desnecessário a utilização de recipientes para armazenamento de água (KUMMU et al., 2016).

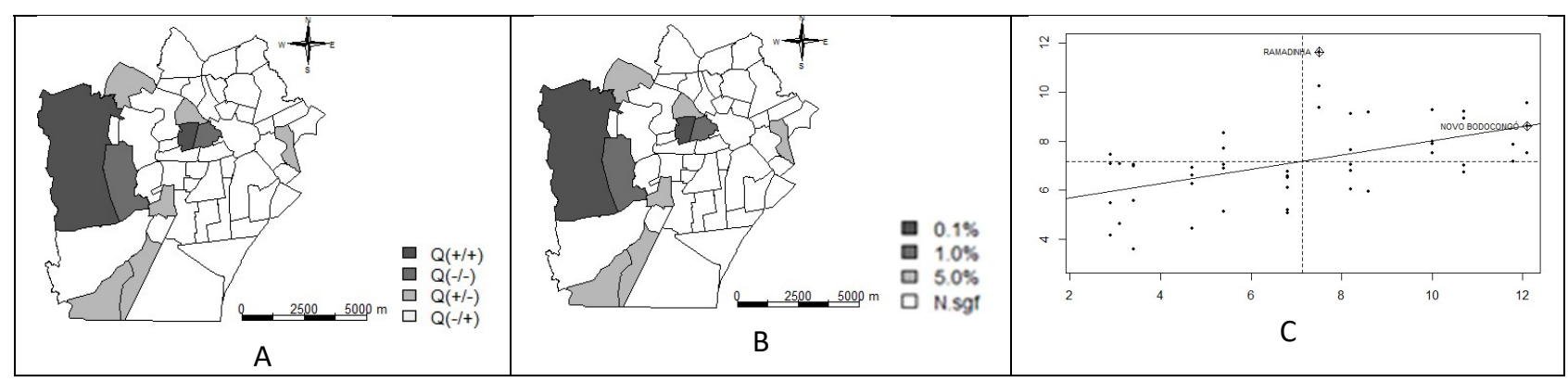

Figura 8: Visualização no Moran Map dos bairros que possuem autocorrelação espacial no 10 LIRAa de 2017(A); ocorrência de bairros significativos apresentados pelo Lisa Map (B); diagrama de espalhamento de Moran (C).

Na Figura 8, têm-se os resultados para 10 LIRAa de 2017. Diante da estatística espacial os bairros: Novo Bodocongó; Serrotão; Malvinas; Santa Cruz e Monte Castelo apresentaram valores positivos e desta forma influenciam para o aumento de focos de larvas do mosquito Aedes aegypti nestes bairros e nos bairros vizinhos. Diante do diagrama de espalhamento de Moran (Figura 8C) o bairro Novo Bodocongó é apontado sendo ainda mais influente. Assim como na análise do Lisa Map (Figura 8B) o bairro do Serrotão apresenta influência positiva para o aumento do índice de LIRAa com 0,1\% percentual de significância.

Na Figura 8A, observa-se os bairros que apresentam valores negativos no 1은 LIRAa de 2017, são eles: Ácacio Figueiredo; Bairro das cidades; Monte Santo; Bela Vista e Prata. Estes bairros estatisticamente contibuem para diminuição dos índices, com destaque para o bairro Bela Vista conforme verificado no Lisa Map (Figura 8B), apresentam-se altamente significativo com o percentual de 0,1\%, exercendo influência negativa e contribuindo para diminuição da incidência de focos nos bairros vizinhos também.

Na Figura 9A, tem-se os resultados para 3은 LIRAa de 2017, com destaque para os bairros: Monte Santo; Prata; Bela Vista; Pedregal e Centenário. Estes bairros apresentam valores negativos e influenciam 
para diminuição dos índices. Conforme também ratificado pelo diagrama de espalhamento de Moram (Figura 8C), a importância dos bairros Centenário, Prata e Bela Vista para diminuição dos focos do mosquito. Sendo ainda mais significativos na análise do Lisa Map os bairros: Pedregal; Bela Vista e Prata (Figura 9B) com $0,1 \%$ de significância. Esse valor expressa a contribuição destes bairros para o ambiente livre de risco de epidemias. Entretanto alguns bairros como: Liberdade; Jardim Paulistano; Tambor; Sandra Cavalcante e Mirante, foram apontados no Moran Map (Figura 9A) com influência espacial positiva influenciando a dispersão de focos do mosquito nos bairros vizinhos.

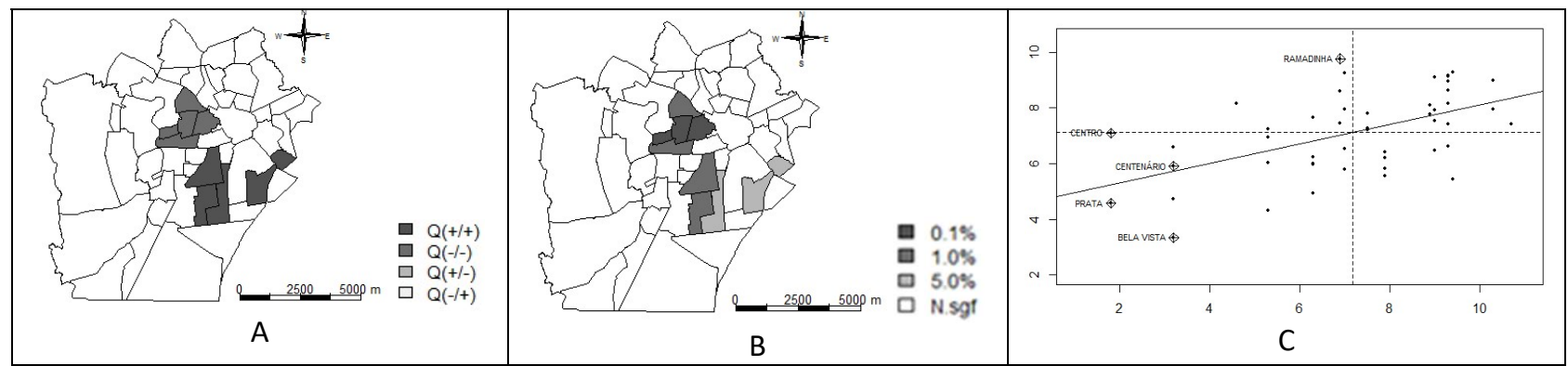

Figura 9: Visualização no Moran Map dos bairros que possuem autocorrelação espacial no 3 LIRAa de 2017 (A); ocorrência de bairros significativos apresentados pelo Lisa Map (B); diagrama de espalhamento de Moran (C).

Nesse sentido, a visualização dessas localidades evidenciam a vulnerabilidade ambiental dessas áreas e a probabilidade da população destes bairros estarem suscetíveis a doenças transmitidas pelo mosquito Aedes aegypti. Vale salientar que o risco de se infectar com os vírus transmitidos pelo mosquito, não é apenas para aqueles que residem em Campina Grande. Tendo em vista que na cidade se concentra um polo universitário com estudantes de várias localidades da Paraíba e a cidade também é referência no Estado em especialidades médicas. A dengue pode se propagar por regiões diferentes daquelas de origem, devido ao fato de uma pessoa que mora em uma localidade sem infestação se contaminar em outras áreas (NASCIMENTO et al., 2015; SOUZA et al., 2012).

A análise espacial dos bairros torna-se uma ferramenta importante, de modo a identificar áreas pontuais prioritárias com influência positiva na proliferação de larvas do mosquito Aedes aegypti em Campina Grande. Contribuindo também para o direcionando de políticas de saúde ambiental auxiliando os gestores na tomada de decisões de vigilância em saúde, tais como na definição de estratégias de controle, prevenção e responsabilização da população favorecendo desta forma a diminuição da incidência larvária (CARVALHO et al., 2018; MEDRONHO, 2017; MAJID et al., 2019; SCANDAR, 2007).

\section{CONCLUSÕES}

A análise da distribuição espacial de larvas do Aedes aegypti na cidade de Campina Grande possibilitou a visualização de bairros que apresentam autocorrelação espacial positiva pela permanência de focos do mosquito na cidade. Fato que resulta da positividade e do uso de recipientes do tipo A2. Verificouse que, um dos principais fatores que contribuíram para propagação das larvas do mosquito nos bairros, caracteriza-se devido a deficiência estrutural do abastecimento e a permanência de depósitos ao nível do solo utilizados para armazenamento de água. Isso revela o quanto à cidade de Campina Grande se tornou 
vulnerável no período em o açude Epitácio Pessoa passou pelo período mais crítico de escassez hídrica de toda sua série histórica, culminando no colapso das águas do manancial.

Neste sentido, observa-se a deficiência do abastecimento público como fator de exposição destes bairros a diferentes graus de risco e maior probabilidade de ocorrência das doença transmitas pelo mosquito Aedes aegypti. Assim, ressalta-se a necessidade de investir na gestão eficiente do uso das águas do açude Epitácio Pessoa, consequentemente garantindo a regularização do abastecimento sem que haja necessidade de estocagem de água. A intensificação das ações educativas de prevenção e controle ao mosquito se tornam importantes em situações de escassez hídrica, para que o risco de epidemias não possa ser agravado, promovendo a redução do índice de infestação do mosquito e consequentemente o risco de novas epidemias.

\section{REFERÊNCIAS}

BRASIL. Boletim Epidemiológico: Vigilância em Saúde. Brasília: MS, 2015.

BRASIL. 0 agente comunitário de saúde no controle da dengue. Brasília: MS, 2009.

BRAGA, A. C. F. M.; GALVÃO, C. O.; BRAGA, C. F. C.. Determinação do padrão de racionamento de água em reservatórios. In: CONGRESSO BRASILEIRO DE ENGENHARIA SANITÁRIA E AMBIENTAL, 21. Anais. João Pessoa: ABES, 2001.

BONITA, R.; BEAGLEHOLE, R.; KJELLSTRÖM,T.. Epidemiologia básica. 2 ed. São Paulo: Nacional, 2010.

BONAT, W. H.; JÚNIOR, P. J. R.; DALLAZUANNA, H. S.; REGIS, L. N.; M ONTEIRO, A. M. V.; SILVEIRA, J. C. ; ACIOLI, R. V.. Investigando fatores associados a contagens de ovos de Aedes aegypti coletados em ovitrampas em Recife/ PE. Revista Brasileira de Biometria, São Paulo, v.27, n.4, p.519537, 2009.

CABRAL, J. A.; FREITAS, M. V.. Distribuição espacial e determinantes socioeconômicos e demográficos da dengue nos municípios brasileiros. Revista Brasileira de Estudos Regionais e Urbanos, v.6, n.1, p.81-95, 2012.

CHUMSRI, A.; TINA, F. W.; JAROENSUTASINEE, M.; JAROENSUTASINEE, K.. Seasons and socio-cultural practices affecting Aedes mosquito larvae in southern Thailand. Tropical Biomedicine, v.35, n.1, p.111-125, 2018.

CARVALHO, S.; MAGALHÃES, M. A. F. M.; MEDRONHO, R. A.. Analysis of the spatial distribution of dengue cases in the city of Rio de Janeiro, 2011 and 2012. Revista Saúde Pública, São Paulo, v.51, n.79, p.1-10, 2017. DOI:

http://dx.doi.org/10.11606/s1518-8787.2017051006239

CONYER, R. T.; GALVÁN, J. M.; ZÚÑIGA, P. B.. Community participation in the prevention andcontrol of dengue: the patio limpio strategy in Mexico.. Paediatrics and International Child Health, v.32, n.1, p.10-13, 2012. DOI: https://doi.org/10.1179/2046904712Z.00000000047

CORTE, R. L.; MELO, V. A. D.; DOLABELLA, S. S.; MARTEIS, L. S...Variation in temephos resistance in field populations of
Aedes aegypti (Diptera: Culicidae) in the State of Sergipe, Northeast Brazil. Revista da Sociedade Brasileira de Medicina Tropical, Uberaba, v.51, n.3, p.284-290, 2018. DOI: http://dx.doi.org/10.1590/0037-8682-0449-2017

DARI, A. L.. Desenvolvimento de um índice de qualidade do serviço de abastecimento de água da cidade de Campina Grande/PB. Dissertação (Mestrado Engenharia Civil e Ambiental) - Universidade Federal de Campina Grande, Campina Grande, 2015.

DALEZIOS, N. R.; ANGELAKIS, A. N.; ESLAMIAN, S.. Water scarcity management: part 1: methodological framework. Global Environmental Issues, v.17, n.1, p.1-40, 2018. DOI: http://doi.org.10.1504/ijgenvi.2018.10011706

DU, S.; LIU, Y.; LIU, J.; ZHAO, J.; CHAMPAGNE, C.; TONG, L.; ZHANG, R.; ZHANG, F.; QIN, F. C.; MA. P.; CHEN,C.H.; LIANG, G.; LIU, Q.; YONG SHI, P.; BERNARD, C.; WANG, P.; TIAN, H.; CHENG, G.. Aedes mosquitoes acquire and transmit Zika virus by breeding in contaminated aquatic environments. Nature Communications, v.10, n.1038, p.1-11, 2019. DOI: https://doi.org/10.1038/s41467-019-09256-0

FERDOUSI, F.; YOSHIMATSU, S.; ENBO, M.; SOHEL, N.; WAGATSUMA, Y.. Identification of Essential Containers for Aedes Larval Breeding to ControlDengue in Dhaka, Bangladesh. Tropical Medicine and Health, v.43, n.4, p.253264, 2015. DOI: https://doi.org/10.2149/tmh.2015-16

GRANDE, M. H. D.; GALVÃO, C. O.; MIRANDA, L. I. B.; SOBRINHO, L. D. G.. A percepção de usuários sobre os impactos do racionamento de água em suas rotinas domiciliares. Ambiente \& Sociedade, São Paulo, v.19, n.1, p.163-182, 2016. DOI: http://dx.doi.org/10.1590/18094422asoc150155r1v1912016

GIL, L. H. S.; KATSURAGAWA, T. H.; LIMA, A. A. de; TADA, M. S.; L. S. OZAKI; JULIÃO, G. R.. Rudimentary cesspits as breeding sites for Aedes aegypti in urban areas of Northern Brazil.. Revista Pan-Amazônica de Saúde, Ananindeua, v.6, n.3, p.73-80, 2015. DOI: http://dx.doi.org/10.5123/S2176$\underline{62232015000300010}$

GONZALEZ, M. F. V.; GRILLET, M.E.; SALAS, Z. I. V.; LIZARAZO, E. F.; AMARISTA, M. A.; SIERRA, G. M.; COMACH, G.; TAMI, 
A.. Spatial analysis of dengue seroprevalence and modeling of transmission risk factors in a dengue hyperendemic city of Venezuela. Neglected Tropical Diseases, p.1-21, 2017. DOI: https://doi.org/10.1371/journal.pntd.0005317

HASIM, M. H.; HIONG, T. G.; MUTALIP, M. H. A.; MAHMUD, M. A. F.; LODZ, N. A.; YOEP,N.; PAIWAI, F.. Spatial density of dengue incidence: $A$ case study of a dengue outbreak in Seksyen 7, Shah Alam. International Journal of Mosquito Research, v.5, n.2, p.9-14, 2018.

HACKL, J.; DUBERNET, T.. Epidemic Spreading in Urban Areas Using Agent-Based Transportation Models. Future InternetMDPI, v.11, n.92, p.1-14, 2019.

IBGE. Instituto Brasileiro de Geografia e Estatística. Dados do censo: população. Rio de Janeiro: IBGE, 2017.

JOHANSEN, I. C.. Urbanização e saúde da população: o caso da dengue em Caraguatatuba (SP). Dissertação (Mestrado em Demografia) - Universidade Estadual de Campinas, Campinas, 2014.

KUMMU, M.; GUILLAUME, J. H. A.; MOEL, H.; EISNER, S.; FLÖRKE, M.; PORKKA, M.; SIEBERT, S.; VELDKAMP, T. I. E.; WARD, P. J.. The world's road to water scarcity: shortage and stress in the 20th century and pathways towards sustainability. Nature, v.6, n.38495, p.1-16, 2016. DOI: http://doi.org.10.1038/srep38495

MEDEIROS, L. E. L.. Utilização dos indicadores convencionais e de satisfação dos usuários para avaliação da qualidade do serviço de abastecimento de água na cidade de Campina Grande/PB. Dissertação (Mestrado em Engenharia Civil e Ambiental) - Universidade Federal de Campina Grande, Campina Grande, 2017.

MAJID, N. A.; NAZI, N. M.; MOHAMED, A. F.. Distribution and Spatial Pattern Analysis on dengue cases in Seremban district, Negeri Sembilan, Malaysia. Sustainability, v.11, n.13, p.2-14, 2019. DOI: http://doi.org.10.3390/su11133572

NEIDERUD, C. J.. How urbanization affects the epidemiology of emerginginfectious diseases. Infection Ecology \& Epidemiology, v.5, n.1, p.1-8, 2015.

NUNES, M. R. T.; FARIA, N. R.; VASCONCELOS, J. M.; GOLDING, N.; KRAEMER, M. U. G.; OLIVEIRA, L. F.; AZEVEDO, R. S. S.; SILVA, D. E. A.; SILVA, E. V. P.; SILVA, S. P.; CARVALHO, V. L.; COELHO, G. E.; CRUZ, A. C. R.; RODRIGUES, S. G.; VIANEZ JR, J. L. S. G.; NUNES, B. T. D.; CARDOSO, J. F.; TESH, R. B.; HAY, S. I.; PYBUS, O. G.; VASCONCELOS, P. F. C.. Emergence and potential for spread of Chikungunya virus in Brazil. BMC Medicine, v.13, n.12, p.1-11, 2015. DOI: https://doi.org/10.1186/s12916-015-0348-x

OLIVEIRA, L. L.. Análise de políticas públicas de saúde: proposição de um modelo de inter-relações complexas aplicado à Política Nacional de Saneamento Básico, no Estado da Paraíba. Tese (Doutorado em Recursos Naturais) Universidade de Federal de Campina Grande, Campina Grande, 2018.
PARAÍBA. Dengue e chikungunya. Boletim Epidemiológico. Paraíba: SSE, 2015.

PARAÍBA. Produto interno bruto dos municípios do Estado da Paraíba. Paraíba: SSE, 2014.

POWELL, J. R.; TABACHNICK, W. J.. History of domestication and spread of Aedes aegypti. Memórias do Instituto Oswaldo Cruz, Rio de Janeiro, v.108, n.1, p.11-17, 2013 2013. DOI: http://dx.doi.org/10.1590/0074-0276130395

RÊGO, J. C.; GALVÃO, C. O.; RIBEIRO, M. M. R.; ALBUQUERQUE, J. P. T. ; NUNES, T. H. C.. A crise do abastecimento de Campina Grande: atuações dos gestores, usuários, poder público, imprensa e população. In: SIMPÓSIO BRASILEIRO DE RECURSOS HÍDRICOS, 21. Anais. Brasília: ABRH, 2015.

ROUQUAYROL, M. Z; ALMEIDA FILHO, N.. Introdução à epidemiologia. 4 ed. Rio de Janeiro: Guanabara Koogan, 2006.

SRINIVASAN, V.; LAMBIN, E. F.; GORELICK, M. S.; THOMPSON, B. H.; ROZELLE, S.. The nature and causes of the global water crisis: Syndromesfrom a meta-analysis of coupled human-water studies. Water Resources Research, v.48, n.10516, p.1-16, 2012. DOI: http://doi.org.10.1029/2011WR011087

SOUZA, L. S.; BARATA, R. C. B.. Diferenciais intraurbanos na distribuição de dengue em Cuiabá, 2007 e 2008. Revista Brasileira de Epidemiologia, São Paulo, v.15, n.4, p.761-770, 2012. DOI: http://dx.doi.org/10.1590/S1415790X2012000400008

SCANDAR, S. A. S.. Análise espacial da distribuição dos casos de dengue e a relação com fatores entomológicos, ambientais e socioeconômicos no município de São José do Rio Preto/SP-Brasil. Tese (Doutorado em Saúde Pública)Universidade de São Paulo, São Paulo, 2007.

SCANDAR, S. A. S.; VIEIRA, P.; JUNIOR, R. P. C.; SILVA, R. A.; PAPA, M.; SALLUM, M. A. M.. Dengue em São José do Rio Preto, Estado de São Paulo, Brasil, 1990 a 2005: fatores entomológicos, ambientais e socioeconômicos. Bepa, v.7, n.81, p.4-16, 2010.

TSUZUKI, A.; HUYNHB, T.; LUU, L.; TSUNODA, T.; TAKAGI, M. Dengue. Effect of water supply system installation on distribution of water storage containers and abundance of Aedes aegypti immatures in urban premises of Ho Chi Minh City, Viet Nam. Dengue Bulletin, v.33, p.161-166, 2009.

YUE,Y.; SUN, J.; LIU, X.; REN, D.; LIU, Q.; XIAO, X.; LU, L.. Spatial analysis of dengue fever and exploration of it senvironmental and socio-economic risk factors using ordinary least squares: a case study in five districts of Guangzhou City, China, 2014. International Journal of Infectious Diseases, v.39, n.48, p.39-48, 2018. DOI: https://doi.org/10.1016/j.ijid.2018.07.023

A CBPC - Companhia Brasileira de Produção Científica (CNPJ: 11.221.422/0001-03) detém os direitos materiais desta publicação. Os direitos referem-se à publicação do trabalho em qualquer parte do mundo, incluindo os direitos às renovações, expansões e disseminações da contribuição, bem como outros direitos subsidiários. Todos os trabalhos publicados eletronicamente poderão posteriormente ser publicados em coletâneas impressas sob coordenação da Sustenere Publishing, da Companhia Brasileira de Produção Científica e seus parceiros autorizados. Os (as) autores (as) preservam os direitos autorais, mas não têm permissão para a publicação da contribuição em outro meio, impresso ou digital, em português ou em tradução. 